\title{
Size and sequence polymorphisms in the glutamate-rich protein gene of the human malaria parasite Plasmodium falciparum in Thailand
}

\author{
Sittiporn Pattaradilokrat ${ }^{1,2^{*}}$ (D), Chawinya Trakoolsoontorn ${ }^{1}$, Phumin Simpalipan ${ }^{1}$, Natapot Warrit ${ }^{1}$,
} Morakot Kaewthamasorn ${ }^{2}$ and Pongchai Harnyuttanakorn ${ }^{1}$

\begin{abstract}
Background: The glutamate-rich protein (GLURP) of the malaria parasite Plasmodium falciparum is a key surface antigen that serves as a component of a clinical vaccine. Moreover, the GLURP gene is also employed routinely as a genetic marker for malarial genotyping in epidemiological studies. While extensive size polymorphisms in GLURP are well recorded, the extent of the sequence diversity of this gene is rarely investigated. The present study aimed to explore the genetic diversity of GLURP in natural populations of $P$. falciparum.

Results: The polymorphic C-terminal repetitive R2 region of GLURP sequences from 65 P. falciparum isolates in Thailand were generated and combined with the data from 103 worldwide isolates to generate a GLURP database. The collection was comprised of 168 alleles, encoding 105 unique GLURP subtypes, characterized by 18 types of amino acid repeat units (AAU). Of these, 28 GLURP subtypes, formed by 10 AAU types, were detected in P. falciparum in Thailand. Among them, 19 GLURP subtypes and 2 AAU types are described for the first time in the Thai parasite population. The AAU sequences were highly conserved, which is likely due to negative selection. Standard Fst analysis revealed the shared distributions of GLURP types among the $P$. falciparum populations, providing evidence of gene flow among the different demographic populations.

Conclusions: Sequence diversity causing size variations in GLURP in Thai $P$. falciparum populations were detected, and caused by non-synonymous substitutions in repeat units and some insertion/deletion of aspartic acid or glutamic acid codons between repeat units. The $P$. falciparum population structure based on GLURP showed promising implications for the development of GLURP-based vaccines and for monitoring vaccine efficacy.
\end{abstract}

Keywords: DNA sequence, Genetic variation, Plasmodium falciparum, Glutamate-rich protein

\section{Background}

The rapid and accurate identification of malaria parasite species is critical for clinical malaria diagnosis and subsequent treatment. A microscopic examination of the blood stage malaria on blood films is the most commonly used method in the laboratory and clinics. This technique is simple, low-cost and easy to perform, but it has many limitations, such as the requirement of high

\footnotetext{
* Correspondence: Sittiporn.P@Chula.ac.th

'Department of Biology, Faculty of Science, Chualongkorn University, 254 Phayathai Road, Bangkok 10330, Thailand

${ }^{2}$ Veterinary Parasitology Research Group, Department of Pathology, Faculty of Veterinary Science, Chulalongkorn University, Bangkok 10330, Thailand
}

parasitemia levels and skills for diagnostic interpretation. More importantly, microscopic examination alone cannot reveal the multiplicity of an infection, which is the feature that occurs in malaria-endemic regions. Molecular tools, such as the polymerase chain reaction (PCR), may be considered as a suitable alternative. A number of PCR-based diagnostic assays have been developed and increasingly adopted for clinical malaria diagnostics $[1,2]$. For identification of malaria species, housekeeping genes have been chosen as molecular targets for developing PCR assays, including the small subunit ribosomal RNA gene in the nuclear genome as well as the 
cytochrome $c$ oxidase subunit 1 gene in the mitochondrial genome [3-6]. Additionally, PCR-based assays can be performed using polymorphic loci, including amplified fragment length polymorphism, microsatellites and antigen-coding genes, facilitating the identification of the parasite strains, the identification of monoclonal vs polyclonal infections, and the differentiation between relapse vs re-infections [7-11]. One of the polymorphic antigen-encoding genes often employed as a key genetic marker for strain identification of the human malaria parasite $P$. falciparum is the glutamate-rich protein (GLURP) gene.

The glutamate-rich protein (GLURP), a $220 \mathrm{kDa}$ antigen expressed throughout the life-cycle of the malaria parasites in a mammalian host $[12,13]$, is localized to the Plasmodium falciparum Pfs 38 protein complex on the merozoite surface, and the protein contains a module of six cysteine residues forming three intramolecular disulphide bonds. The Pfs38 complex binds to host erythrocytes directly via glycophorin A as a receptor [14]. The GLURP protein consists of the three defined regions of an N-terminal non-repetitive region (R0), a central repetitive sequence (R1) and a C-terminal repetitive region ( $\mathrm{R} 2)$. The $\mathrm{R} 0$ region is predicted to be hydrophobic and may act as a signal peptide, which is a feature of secretory proteins [13].

Evidence that supports the role of GLURP as an immune target comes from several studies. First, immunizing mice with a vaccinia virus-expressed GLURP of $P$. falciparum induced a humoral immune response [15]. To further map the immunodominant region of GLURP, four purified polypeptides, including the fulllength protein and the R0, R1 and R2 regions, were produced in Escherichia coli and then tested for reactivity with sera from adults living in endemic areas in Liberia. The antibody reactivity against the $\mathrm{R} 2$ and $\mathrm{R} 0$ regions was observed in nearly $100 \%$ of the serum samples, suggesting a role for the $\mathrm{R} 2$ and $\mathrm{R} 0$ regions as the immune target [16]. Subsequent epidemiological studies performed in endemic areas in Africa and South America consistently showed that malaria-infected patients had high levels of antibodies that react specifically with GLURP, and the levels of anti-GLURP antibodies against the $\mathrm{R} 0$ and $\mathrm{R} 2$ regions were positively associated with a reduced risk of clinical malaria [17-21]. Anti-GLURP antibodies against the $\mathrm{R} 0$ region act in synchronization with monocytes or mediate opsonic phagocytosis, conferring protection against febrile malaria [22, 23]. Together, these studies demonstrate that GLURP R0 and R2 may participate in eliciting protective immunity in humans, thereby giving further impetus for the development of malaria vaccines targeting GLURP.

Accordingly, P. falciparum GLURP has currently been employed in vaccine development. One example is the
GMZ2 vaccine, which combines the immunodominant GLURP R0 region and MSP-3 of P. falciparum. The vaccine is designed to mimick naturally acquired antimalarial immunity [24]. In pre-clinical studies, the vaccine appeared to be safe and induced strong protective immune responses against the malaria parasites in simian models $[25,26]$. In Phase 1 clinical trials, GMZ2 was safe, well tolerated and immunogenic in both children and adults [27-29]. Serological analyses of the antibody levels showed high levels of specific and functional antibodies in vaccinated subjects, with the capacity to control the parasite multiplication [30, 31]. Following these trials, a Phase 2 clinical trial was conducted in participants of 1 to 5 years of age in four African countries, and the GMZ2 vaccine was confirmed to be well tolerated, immunogenic and capable of reducing the malaria incidence, but the efficacy was only moderate [32]. It is likely that the efficacy of the vaccine was compromised by the parasite antigenic diversity [33]. Therefore, it is necessary to investigate the extent of genetic polymorphism of any vaccine candidate for malaria parasites in the natural populations where the vaccine is to be used.

The GLURP gene of $P$. falciparum is located on chromosome 10 and encodes a long polypeptide of 1271 amino acids (data of strain F32 from Tanzania) [12, 13]. The repetitive sequences of the R2 region of GLURP are highly conserved, but the number of repeat amino acid units (AAUs) varies among $P$. falciparum isolates, resulting in a size polymorphism. Hence, GLURP is routinely employed in PCR assays as a genetic tool for parasite identification and studying the multiplicity of malaria infections [34-39]. While the size polymorphism of the GLURP R2 region in P. falciparum populations is well reported, surprisingly, there is limited information on the sequence variation that underlines the polymorphisms in GLURP. To date, most of the available GLURP $\mathrm{R} 2$ region sequences (and their translated GLURP amino acid sequences) deposited at the NCBI nucleotide database are derived from $P$. falciparum isolates in India $[34,35]$, with a few unpublished sequences from $P$. falciparum from Iran, Indonesia and China. However, there are no reports of GLURP sequences in P. falciparum populations in Thailand. To address this deficiency, this study aimed to describe the detailed molecular variation in the GLURP R2 region. We generated GLURP sequences from 65 parasite isolates from five representative malaria hotspots in Thailand and combined them with the GLURP sequence data from P. falciparum isolates worldwide, thereby generating a global catalogue of GLURP and, after in silico translation, GLURP. This collection of GLURP sequences can be used to investigate the basis of molecular variations and the signature of natural selection as well as to infer the population structure of P. falciparum. 


\section{Methods}

Amplification and DNA sequencing of the GLURP R2 region The DNA templates for the PCR amplification of GLURP were extracted from $72 \mathrm{P}$. falciparum samples collected from five localities (Mae Hong Son, Kanchanaburi, Ranong, Trat and Ubon Ratchatani) in Thailand, as previously described [10], and shown in Additional file 1: Figure S1. The species of malaria parasites were confirmed by microscopic examinations of the thin blood film and by genotyping with the genetic markers of merozoite surface protein-1 $(m s p-1)$ and $m s p-3$, as previously reported $[40,41]$. For amplification of the R2 region of the GLURP gene, the nOF-primer (forward: 5'-CAA CCA AAT CCA CAA GAA CCA GTT GA-3') and the R2-primer (reverse: 5'-TCT GGT TTA GTG GAT TCA CCT TCA GAT-3') primer pair were used to yield sequences that corresponded to nucleotide positions 2215-2240 and 3441-3467 of the full-length GLURP gene of P. falciparum strain F32 (GenBank: M59706.1) [12]. The total PCR reaction volume $(50 \mu \mathrm{l})$ contained $200 \mu \mathrm{M}$ of dNTPs, $2 \mathrm{mM}$ of $\mathrm{MgCl}_{2}, 0.3 \mu \mathrm{M}$ of each primer, 200-300 ng of parasite DNA and 2.5 units of Taq polymerase enzyme in $1 \mathrm{X}$ Taq PCR buffer (Biotechrabbit, Hennigsdorf, Germany). The PCR conditions started with $95{ }^{\circ} \mathrm{C}$ for $5 \mathrm{~min}$, followed by 40 cycles of $95{ }^{\circ} \mathrm{C}$ for $30 \mathrm{~s}, 65^{\circ} \mathrm{C}$ for $30 \mathrm{~s}$ and $72{ }^{\circ} \mathrm{C}$ for $90 \mathrm{~s}$, and then followed by a final $72{ }^{\circ} \mathrm{C}$ for $5 \mathrm{~min}$. The PCR products were analysed by standard agarose gel electrophoresis in a $2 \%(w / v)$ agarose-TBE gel. The quality of the PCR products was determined under UV transillumination after exposing the gels to ethidium bromide for DNA staining. All the positive PCR products produced a single band and were submitted for commercial automatic DNA sequencing using the Big Dye Terminator System (Applied Biosystems, California, USA) on an ABI3730XL DNA analyser. DNA sequencing was performed twice for each PCR fragment. The sequencing primers included the above PCR primer pair and the additional Int-3 primer (5'-GAA GTG GCT CAT CCA GAA ATT GTT GAA-3'). A total of 65 sequences were unambiguous and included in the present analysis.

\section{Retrieval of GLURP sequences from the NCBI database}

A total of 89 nucleotide sequences of the $\mathrm{R} 2$ region of the GLURP gene from India $(n=81)$, Indonesia $(n=3)$, Iran $(n=2)$ and China $(n=1)$ and the laboratory strains 3D7 and F32 were retrieved from the NCBI nucleotide database (https://www.ncbi.nlm.nih.gov/nuccore) (see Additional file 2: Table S1). In addition, a $\mathrm{BLAST}_{\mathrm{N}}$ search was performed using the nucleotide sequence of the P. falciparum reference strain 3D7 [42] to search against 36 whole-genome shotgun contigs of $P$. falciparum laboratory strains and wild isolates that are deposited in the NCBI database. The whole-genome shotgun contigs with the complete GLURP R2 region were identified in the sequences from $P$. falciparum strains HB3, Dd2, FCC-2/Hainan and VS/1, which were submitted by the Broad Institute (Massachusetts, USA), and a further 10 sequences were from $P$. falciparum isolates from Mali (Africa), which were submitted by the Institute for Genome Sciences (Maryland, USA). Taken together, all the GLURP R2 region sequences from the 103 P. falciparum isolates worldwide and those from the $65 \mathrm{P}$. falciparum Thai isolates in Thailand formed a global database comprised of 168 alleles of the GLURP gene.

\section{Sequence analysis and population genetics analysis}

The GLURP nucleotide sequences were aligned and then manually adjusted, where necessary, using the BioEdit program [43]. Sequence alignment was performed using ClustalW software [44]. The pairwise interpopulation indices or the Wright Fst indices were calculated using the Arlequin software [45]. Other molecular indexes, including the nucleotide diversity $(\pi)$, haplotype index $(h d)$, and neutrality tests (Tajima's $D$ test, Fu and Li's $D^{*}$ test and $\mathrm{Fu}$ and Li's $F^{*}$ test) and linkage disequilibrium, were calculated using the DnaSP software $[46,47]$.

\section{Analysis of the AAU composition and GLURP subtypes}

Theoretical (in silico) translations of the GLURP nucleotide sequences were performed using BioEdit software [43], and the resulting GLURP amino acid sequences were exported into a text format. The published amino acid repeat units (AAU) of blocks $1-15$ in the $\mathrm{R} 2$ region were used to search against the collection of GLURP sequences in order to identify any novel AAU types [34, 35]. A total of 10 AAU types were previously identified between blocks $1-15$ of the GLURP R2 region, including one AAU type $\left(^{*}\right)$ in block 1 , two AAU types (I and II) in block 2, one AAU type (1) in block 3 and 10 AAU types (a, b, c, d, e, f, g, h, i and j) in blocks 4-15 [34, 35]. The arrangements of the AAUs were manually inspected to identify the unique AAU arrangement (GLURP subtype). When novel subtypes were identified, the sequence was BLAST $_{P}$ searched in the NCBI database for confirmation. The total number of AAU types in each block and the parasite isolates with a unique AAU arrangement were recorded. The percentage of AAU types was calculated by dividing the number of a particular AAU type by the total number of AAU types in one block. The diversity of infection (DOI) was calculated by dividing the number of different parasite genotypes that were detected with the total number of parasites that were genotyped [48].

\section{Results}

Nucleotide diversity and neutrality test

The GLURP R2 region was amplified from the genomic DNA of 72 isolates of $P$. falciparum collected from five 
localities in Thailand. A total of 69 PCR products with single PCR amplicons were submitted for DNA sequencing, generating 65 unambiguous nucleotide sequences. The nucleotide sequences of GLURP in P. falciparum from Thailand were then combined with 103 sequences from the NCBI nucleotide collections and from wholegenome shotgun sequences to generate a global collection of 168 GLURP alleles and the in silico translated GLURP alleles in $P$. falciparum isolates worldwide (Additional file 2: Table S1), encoding a total of 105 GLURP subtypes (unique amino acid sequences). The average pairwise nucleotide diversity per site $(\pi)$ and the haplotype index $(h d)$ for all 168 GLURP samples was $0.00777 \pm 0.00066$ and $0.757 \pm 0.031$, respectively (Table 1). The sequence diversity analysis also indicated that the level of genetic diversity in GLURP in the P. falciparum populations in Thailand was higher than in other populations in Asia but was lower than the parasite population in Africa (Mali) (Table 1). Therefore, these results revealed different levels of sequence diversity in GLURP in different demographical locations.

Three neutrality tests (Tajima's $D, F u$ and Li's $D^{*}$ and $\mathrm{Fu}$ and Li's $F^{*}$ statistics) were performed to examine for any evidence of natural selection in terms of estimating the deviation from neutrality, which is based on the expectation of a constant population size at mutationdrift equilibrium $[49,50]$. As shown in Table 1 , no departure from neutrality was detected in any of the populations by the three tests. The negative Tajima's $D$ test signified an excess of low frequency polymorphisms relative to the expected level [49]. Similarly, sliding window analyses of the three neutrality tests revealed non-significant values for the GLURP R2 region in all three tests (Fig. 1). Therefore, the sequence polymorphisms in the GLURP R2 region were apparently neutral, which was likely to be due likely to negative selection.

Furthermore, when linkage disequilibrium analysis was performed on the GLURP R2 region, it revealed a decline in the number of significant linkage disequilibrium between pairs of polymorphic loci (both $R^{2}$ and $D^{\prime}$ ) over molecular distance (Fig. 2), indicating intragenic recombination in the GLURP R2 region. This notion was supported by the presence of five minimum recombination sites, as estimated by means of the four-gamete test (parameter $R_{m}$ ). The recombination parameter between adjacent nucleotide sites and for the whole sequence had a value of 0.0129 and 9.2, respectively, which indicates that the meiotic recombination can contribute to the genetic diversity of the GLURP gene.

\section{Composition of the AAUs in the GLURP R2 region}

The R2 region of GLURP contained 6-15 blocks of AAUs, which could be classified into 18 types. Sequence alignments of the AAU in each block revealed the conserved polymorphic sites and specific distributions of the 18 AAU types in the R2 region (Additional file 3: Table S2).

Blocks 1 and 3 contained single AAU types (* and 1), which were identical in all the $P$. falciparum isolates. Block 2 was characterized by three AAU types (I, II and III), where type I was the most common type, representing $96 \%$ of the block 2 sequence. The AAU types I and II, previously reported in P. falciparum populations in India [34, 35], were also detected in the P. falciparum populations in Thailand, while AAU type III was novel and was detected in the whole-genome shotgun sequences of $P$. falciparum isolate 309 from Mali (Additional file 2: Table S1). The other 13 AAU types were located between blocks 4-15. Of these, three AAU types ( $\mathrm{a}, \mathrm{b}$ and $\mathrm{c}$ ), were common in all the P. falciparum isolates. In contrast, the other ten AAU types (d, e, $\mathrm{f}, \mathrm{g}$, $\mathrm{h}, \mathrm{i}, \mathrm{j}, \mathrm{k}, \mathrm{l}$ and $\mathrm{m}$ ) were relatively less common, and their distributions were reserved in specific blocks. Of these, six AAU types (d, e, f, g, h and i) were previously reported in P. falciparum populations in India [34, 35], while three others (i, $j$ and $\mathrm{l}$ ) were detected in the

Table 1 Summary of statistics for the GLURP R2 region in P. falciparum populations and tests for departure from neutrality

\begin{tabular}{llllllll}
\hline Population & $n$ & GLURP subtype & $h d \pm \mathrm{SD}$ & $\pi \pm \mathrm{SD}$ & Tajima's $D$ & Fu and Li's $D^{*}$ & Fu and Li's F* \\
\hline Thailand & 65 & 28 & $0.833 \pm 0.038$ & $0.00611 \pm 0.00058$ & $-0.53408(P>0.10)^{\mathrm{a}}$ & $0.80029(P>0.10)$ & $0.38828(P>0.10)$ \\
India & 81 & 68 & $0.944 \pm 0.016$ & $0.00377 \pm 0.00103$ & $-0.29581(P>0.10)$ & $-0.88322(P>0.10)$ & $-0.79358(P>0.10)$ \\
Indonesia & 3 & 3 & $1 \pm 0.272$ & $0.00102 \pm 0.00064$ & nd & nd & nd \\
Iran & 2 & 1 & 0 & 0 & nd & nd & nd \\
China & 2 & 2 & $1 \pm 0.050$ & $0.00159 \pm 0.00079$ & nd & nd \\
Mali & 10 & 10 & $1 \pm 0.045$ & $0.00915 \pm 0.00219$ & $-0.70120(P>0.10)$ & $-0.73515(P>0.10)$ & $-0.81915(P>0.10)$ \\
Laboratory lines & 5 & 5 & $1 \pm 0.126$ & $0.01170 \pm 0.00439$ & $-0.29817(P>0.10)$ & $-0.29817(P>0.10)$ & $-0.31445(P>0.10)$ \\
Global population & 168 & $105^{b}$ & $0.757 \pm 0.031$ & $0.00777 \pm 0.00066$ & $-0.13448(P>0.10)$ & $-2.05874(P>0.10)$ & $-0.21350(P>0.10)$
\end{tabular}

Abbreviations: $n$ number of sequences, $h d$ haplotype diversity index, $\pi$ nucleotide diversity index, $S D$ standard deviation, nd neutrality tests not determined as the minimum number of sequences required for neutrality tests is four

${ }^{a}$ Statistical significance was deemed at a $P$-value of $<0.05$

${ }^{\mathrm{b}}$ Total number of GLURP subtypes in the global population is lower than the sum of GLURP subtypes in each population because some GLURP subtypes are detected in more than one population 

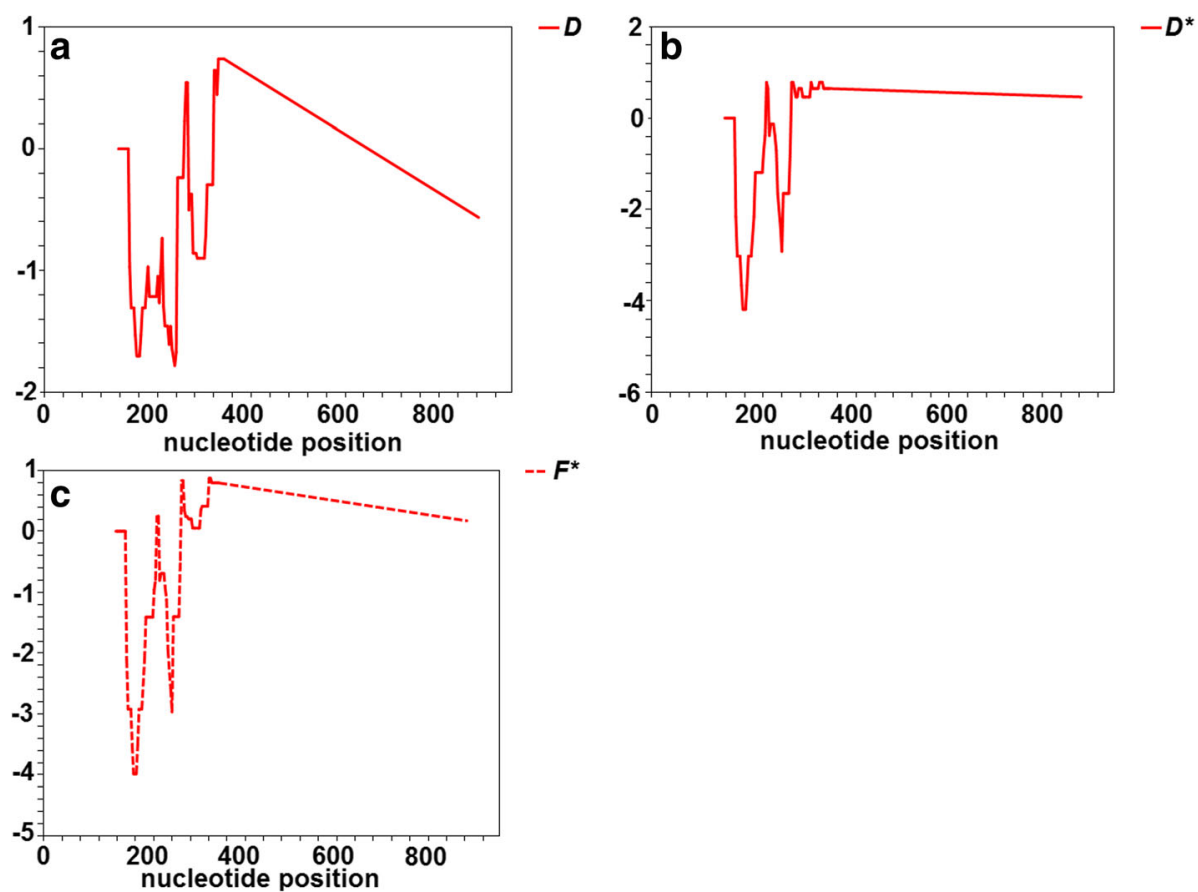

Fig. 1 Sliding window plots of the Tajima $D^{\prime}(\mathbf{a})$, Fu and Li's $D^{*}(\mathbf{b})$ and Fu and Li $F^{*}$ values (c) for the R2 region of the GLURP gene of P. falciparum. Nucleotide numbers are from the start site of the GLURP R2 region. Window length is $25 \mathrm{bp}$, and the step size is $3 \mathrm{bp}$. All values were statistically insignificant

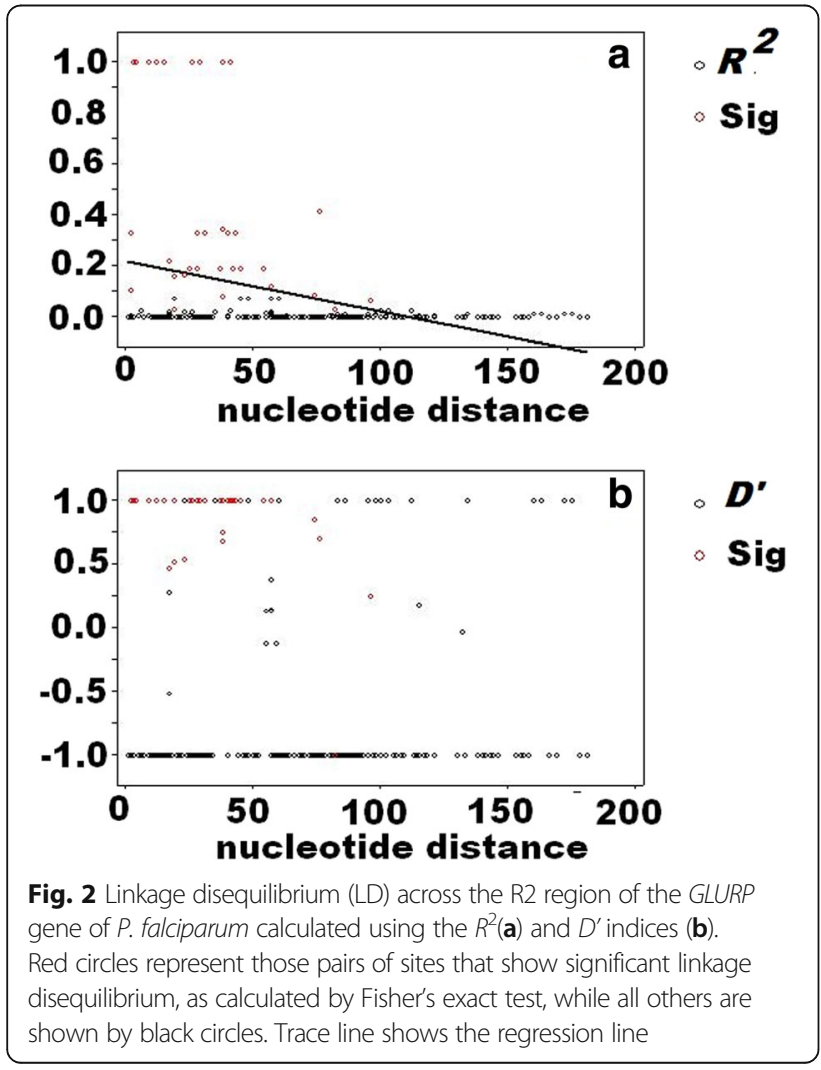

whole-genome shotgun sequences of the P. falciparum populations in Mali (Additional file 2: Table S1).

Analysis of the GLURP R2 region in the Thai isolates of P. falciparum identified a total of 13 AAU types, including one AAU type in block $1(*)$, two AAU types in block 2 (I and II), one AAU type in block 3 (1) and six AAU types (a, b, c, k, l and $\mathrm{m}$ ) between blocks 4-14. Of these, two AAU types ( $\mathrm{k}$ and $\mathrm{m}$ ) were described for the first time in the $P$. falciparum populations in Thailand. Specifically, AAU type k was located in block 5 in the P. falciparum isolates RN130 and RN133, while AAU type $m$ was detected in block 12 in the P. falciparum isolates RN72 and RN122. These parasite isolates were all from the same population in Ranong Province.

\section{Size polymorphism in the GLURP R2 region}

The GLURP R2 region with a different number of repeat units was detected at different frequencies in the $P$. falciparum populations studied (Table 2). The GLURP with 11 repeat units was the most common GLURP allele, being detected in $53 \mathrm{P}$. falciparum worldwide isolates (31\%). In contrast, GLURP with extremely short (6) or long (15) repeat units appeared to be extremely rare. It should also be noted that GLURP with 13 repeat units had the highest allelic diversity, with a total of 26 GLURP subtypes being identified, representing 25\% of the total AAU pattern variations (Table 2). In contrast, GLRUP with 6, 8 and 15 repeat units exhibited a lower 
Table 2 Frequency, subtypes and size of the $P$. falciparum GLURP polypeptide in global database. Size indicates the number of amino acid residues detected between blocks 4-15

\begin{tabular}{lllll}
\hline No. of repeat units & $n$ & GLURP subtype & Subtype name & $\begin{array}{l}\text { Size } \\
\text { (aa residue) }\end{array}$ \\
\hline 6 & 1 & 1 & A1 & 57 \\
8 & 3 & 2 & C1 to C2 & $97-98$ \\
9 & 10 & 4 & D1 to D4 & $115-117$ \\
10 & 27 & 19 & E1 to E19 & $133-137$ \\
11 & 53 & 22 & F1 to F22 & $152-156$ \\
12 & 16 & 14 & G1 to G14 & $172-176$ \\
13 & 37 & 26 & H1 to H26 & $191-195$ \\
14 & 19 & 15 & I1 to I15 & $210-214$ \\
15 & 2 & 2 & J1 to J2 & $229-235$ \\
\hline
\end{tabular}

Note: The variation in size in blocks 4-15 is due to the insertion/deletion of aspartic acid and glutamic acid between the blocks. The numbers of amino acid residues in blocks 1-3 are invariable (block 1, 20 amino acid residues; block 2, 32 amino acid residues; block 3, 18 amino acid residues). See Additional file 2 for the AAU arrangement of the GLURP subtypes

Abbreviations: aa amino acid, $n$ number of parasites

genetic diversity, with 1, 2 and 2 identified subtypes, respectively.

In addition to the variation in the numbers of repeat units, the size polymorphism of the $\mathrm{R} 2$ region of GLURP resulted from the insertion or deletion of negatively charged amino acids, including aspartic acid (D) and glutamic acid (E), between the repeat units. The addition of aspartic acid was more frequently detected between blocks 4-9, while the addition of glutamic acid appeared more frequent between blocks 10-12 (Additional file 4: Table S3). Taken together, these results indicated that variations in the repeat units and the insertions/deletions of aspartic acid and glutamic acid between the repeat units contributed to the size polymorphism in GLURP.

\section{Distribution of the GLURP subtypes and the population structure of $P$. falciparum}

The analysis conducted using the 65 alleles of the GLURP R2 region from $P$. falciparum populations in Thailand identified 28 GLURP subtypes, containing 8-14 repeat units (Additional file 5: Table S4). The majority (41\%) of these Thai P. falciparum isolates inherited GLURP with 11 repeat units. This is contradictory to a previous analysis of GLURP sequences from Indian populations [34, 35], where the GLURP with 13 repeat units was the most common. The DOI analysis of the Thai $P$. falciparum populations revealed different levels of genetic diversity of GLURP among the Thai subpopulations of $P$. falciparum, where the DOIs of $P$. falciparum at Mae Hong Son, Kanchanaburi and Ranong (Western Thailand) were 0.60, 0.76 and 0.56, while those at Ubon Ratchatani and Trat (Eastern Thailand) were both 0.83. The implication then is that the parasite subpopulations near eastern Thailand were more genetically diverse than those near western Thailand.

Of the Thai GLURP subtypes, the F8 and F11 subtypes were the most common, being identified in 13 and nine P. falciparum isolates, respectively, representing 37\% of the parasite populations in Thailand (Additional file 5: Table S4). Interestingly, the D3 subtype matched the GLURP R2 sequence of $P$. falciparum isolate 2/GL from Indonesia (Eisen et al., unpublished data), while eight other subtypes (D4, E9, F8, F11, H1, H10, H22 and I5) corresponded to the GLURP sequences of $P$. falciparum isolates from India $[34,35]$. It is also noteworthy that the F11 subtype was identical to the sequence of the $P$. falciparum reference strain $\mathrm{Dd} 2$. However, no parasite isolates in Thailand or worldwide had GLURP sequences that were similar to those of strains 3D7 and F32, which were employed in the genome sequencing project [42] and the production of the GLURP-based malaria vaccine, respectively [16]. As a result, the present study identified 19 novel GLURP subtypes in Thailand.

The distribution analysis showed that approximately one-third of the GLURP subtypes were shared among the Thai $P$. falciparum subpopulations (Additional file 5: Table S4). To further determine the genetic homogeneity between the parasite populations, pairwise interpopulation comparisons were performed for each parasite population using Wright's fixation index $(F s t)$. The Fst values from all pairs of the $P$. falciparum populations were low and non-significant (Table 3), indicating a similar distribution of the GLURP subtypes in the five $P$. falciparum populations in Thailand. Thus, gene flow might have operated in $P$. falciparum subpopulations from the Thailand-Myanmar, Thailand-Laos and Thailand-Cambodia borders. In addition, the Fst index for the GLURP alleles from $P$. falciparum in Thailand and India $(n=81)$ was $-0.00312(P=0.24)$. This low and non-significant Fst value indicated that there was genetic

Table 3 Pairwise $F_{\text {st }}$ indices of the GLURP alleles between $P$. falciparum populations in Thailand. Fst values were low and non-significant in all pairs of $P$. falciparum populations

\begin{tabular}{lllll}
\hline & $\mathrm{K}$ & $\mathrm{MH}$ & $\mathrm{RN}$ & $\mathrm{TD}$ \\
\hline $\mathrm{MH}$ & -0.00499 & - & & \\
& $(P=0.40)$ & & & \\
$\mathrm{RN}$ & 0.02430 & -0.58080 & - & - \\
& $(P=0.23)$ & $(P=0.81)$ & & \\
TD & -0.04670 & -0.06146 & 0.00448 & - \\
& $(P=0.80)$ & $(P=0.68)$ & $(P=0.32)$ & \\
UB & 0.07521 & 0.05245 & 0.07187 & 0.07195 \\
& $(P=0.11)$ & $(P=0.16)$ & $(P=0.10)$ & $(P=0.14)$ \\
\hline
\end{tabular}

Abbreviations: $M H$ Mae Hong Son, $K$ Kanchanaburi, RN Ranong, TD Trat, UB Ubon Ratchatani 
homogeneity in the GLURP subtypes between these $P$. falciparum populations in Thailand and India.

\section{Discussion}

The present study reports the genetic structure and diversity of the immunodominant GLURP R2 region in $P$. falciparum populations in Thailand and worldwide. As of September 2017, 103 sequences of the GLURP gene were available in the NCBI database. Sequence analysis led to the identification of 86 unique GLURP subtypes. In the present study, 65 GLURP sequences were generated from $P$. falciparum populations in Thailand, and 28 GLURP subtypes were identified, including 19 new subtypes that were identified for the first time. Overall, the combined database of GLURP revealed 105 GLURP subtypes in 168 alleles of the $P$. falciparum isolates worldwide.

Variations in the repeat unit numbers led to size polymorphisms in the GLURP R2 region. In the present study, GLURP, with 8-14 repeat unit blocks was detected in $P$. falciparum in Thailand, with 11 repeat unit blocks being the most frequent. This result contrasts with previous analyses of GLURP sequences from P. falciparum in Indian isolates [34, 35], where GLURP genes with 9-15 repeat unit blocks were detected, with 13 repeat units being the most common. Within the whole-genome shotgun sequences of $P$. falciparum laboratory strains, the shortest GLURP R2 region was found to contain only six repeat units, which was detected in P. falciparum HB3. Overall, the data demonstrated the extensive size variation in the GLURP gene in both laboratory strains and natural isolates of $P$. falciparum.

Comparison of the amino acid repeat units revealed conserved amino acid substitutions amongst the repeat units. Of the 18 AAU types identified, the majority were located between blocks 4-15, with fewer AAU types in blocks 1-3. Indeed, blocks 1 and 3 had identical sequences in all $P$. falciparum isolates. The limited amino acid substitutions in the AAU likely reflects the functional constraints of the GLURP protein. A recent study reported that GLURP formed the Pfs 38 protein complex on the merozoite surface that binds to erythrocytes via glycophorin A and participates in the parasite invasion of erythrocytes [14]. This is consistent with the three neutrality tests (Tajima's $D, \mathrm{Fu}$ and Li's $D^{*}$ and $\mathrm{Fu}$ and Li's $F^{*}$ tests) that all gave non-significant values, suggesting that the conserved AAU sequences were maintained under negative purifying selection. Together, these results indicated that the AAU sequences of GLURP are highly conserved, supporting the view that GLURP is an attractive target for vaccine development [17-21].
Another important finding in the present study was that the GLURP R2 region in Thai populations of $P$. falciparum exhibited extensive sequence variation, with a total of 13 AAU types and 28 GLURP subtypes. The majority of these GLURP subtypes (19 subtypes, 67\%) in Thailand were novel. However, the number of GLURP subtypes in P. falciparum in Thailand was lower than that in Indian isolates, where 61 GLURP subtypes were seen $[34,35]$. This contrasts to a previous genetic analysis of $P$. falciparum using $m s p-3$ gene sequences, in which fewer $m s p-3$ haplotypes were detected in the $P$. falciparum populations in India than in Thailand [41]. Accordingly, the genetic diversity in $P$. falciparum populations should be evaluated using multiple genetic loci. If genetic markers that exhibit low or limited polymorphisms are employed for genotyping, it is likely that the genetic variation of parasite populations will be underestimated. Because GLURP is highly polymorphic in size and is not under the influence of positive selection, this marker would be suitable for epidemiological and population genetic studies.

The mechanisms of the molecular variation in GLURP can be summarized as follows. First, the emergence of the novel GLURP subtypes was attributed to nonsynonymous amino acid substitutions in different repeat units that resulted in the formation of new AAU types. Of the 18 AAU types identified in P. falciparum worldwide, two AAU types ( $\mathrm{l}$ and $\mathrm{m}$, see Additional file 3: Table S2) are reported here for the first time. In addition, novel GLURP subtypes might have arisen from genetic recombination, resulting in a new assortment of AAU types, as well as by the insertion and deletion of extra amino acids, including aspartic acid (D) and glutamic acid (E), between the repeat units. Taken together, our study revealed the mechanisms contributing to the genetic diversity of the GLURP gene in P. falciparum populations.

Comparison of GLURP subtypes within the Thai $P$. falciparum populations revealed different levels of genetic diversity among the different $P$. falciparum populations. From the DOI indices, $P$. falciparum populations in eastern Thailand were more diverse than those in western Thailand. The low DOI simply reflected that the $P$. falciparum populations in Mae Hong Son, Kanchanaburi and Ranong had large numbers of individuals with the same GLURP subtypes. The analysis also showed that different subpopulations of $P$. falciparum in Thailand shared a large number of GLURP subtypes, indicating that gene exchange may occur between the parasites near the eastern and western borders of Thailand. This hypothesis was supported by the Wright's statistical analysis, where low and non-significant Fst values were found for all the pairs of compared P. falciparum populations. In addition, the Fst analysis of the GLURP 
subtypes in P. falciparum in India and Thailand also showed a low and non-significant Fst value, indicating similar GLURP alleles between the parasite populations. Together, these results demonstrated that $P$. falciparum populations in different geographical locations may exhibit different levels of genetic diversity, but gene flow likely exists among the P. falciparum populations in the Greater Mekong Subregion and India.

The sequence analysis and population structure presented here may be useful for developing future GLURP-based vaccines and for monitoring vaccine efficacy. Currently, the GMZ2 vaccine, which incorporates the GLURP R0 region of $P$. falciparum strain F32 and MSP-3, is being tested in clinical studies in various endemic areas $[32,51]$. Although GMZ2 reduced the malaria incidence, its efficacy was still low and so vaccines using a more immunogenic formulation are needed. One proposed strategy is to incorporate the GLURP R2 region of $P$. falciparum strain Dd2 in a vaccine, since the R2 region also contains B-cell epitopes and might elicit functional protective antibodies [52], while the most common GLURP subtype (F11) in Thailand and India is identical to that of Dd2. Thus, such a vaccine might exhibit improved efficacy against clinical cases of malaria.

\section{Conclusions}

The present study generated a database of GLURP gene sequences, and the in silico translated GLURP sequences, from worldwide populations of P. falciparum. The database revealed not only the sequence diversity but also the distribution of the GLURP subtypes. Because the GLURP gene exhibited extensive DNA size and sequence polymorphism, it might serve as a genetic marker for parasite identification as well as for population and epidemiological studies. The sequence information provides knowledge for a better design of GLURP-based malaria vaccines and reveals the population structure of $P$. falciparum across Thailand, which is of use for monitoring and controlling this parasite.

\section{Additional files}

Additional file 1: Figure S1. Map showing the sampling site locations for Plasmodium falciparum collection in Thailand. Abbreviations: $\mathrm{MH}$, Mae Hong Son; K, Kanchanaburi, RN, Ranong; UB, Ubon Ratchatani; TD, Trat. (DOC $67 \mathrm{~kb}$ )

Additional file 2: Table S1. The AAU arrangement in 168 alleles of GLURP in P. falciparum isolates worldwide. The capital letters D and $E$ represents the additions of aspartic acid and glutamic acid, respectively, between the repetitive regions (blocks 4-14). The lowercase letters in blocks 4-14 indicate the amino acid repeat unit (see also Additional file 3: Table S2). (XLS $133 \mathrm{~kb}$ )

Additional file 3: Table S2. Amino acid sequences and frequency (in $\%$ ) of AAUs in the R2 region of the GLURP gene in $P$. falciparum. AAUs were named according to the GLURP sequences of $P$. falciparum in India
[34,35]. Bold letters indicate the amino acid substitutions that define AAU type (XLS $43 \mathrm{~kb}$ )

Additional file 4: Table S3. The percentage of the aspartic acid (D) and glutamic acid (E) insertions at the end of Block 4 to 15 sequences in R2 region of $P$. falciparum GLURP gene. (DOC $34 \mathrm{~kb}$ )

Additional file 5: Table S4. Distribution of the GLURP subtypes in 65 P. falciparum isolates in Thailand. Bold letters indicate the 10 GLURP subtypes that are prevalent in more than one endemic site in Thailand (DOC 92 kb)

\section{Acknowledgements}

We are grateful to Dr Robert Butcher for language editing and proofreading the manuscript. We would like to thank the editor and reviewers for their insightful comments and suggestions on the paper, as these comments led us to an improvement of the work.

\section{Funding}

This work was supported by grants from the Development and Promotion of Science and the Technology Talents Project (DPST) graduate with First Placement, Royal Thai Government to SP. The sponsor of the study had no role in the study design, data collection, data analysis, data interpretation or writing of the manuscript.

\section{Availability of data and materials}

The nucleotide sequence data of the different GLURP genes reported in this paper have been submitted to the GenBank database with accession numbers MG578444-MG578508. The data are also available upon request. Requests for data may be made by contacting SP.

\section{Authors' contributions}

SP and PH provided the conceptual framework for the project, guidance for the interpretation of the data, performed the data analysis and participated in the manuscript preparation, revision and coordination. SP, CT and PS performed the molecular genetic work. SP, NW and MK analysed the data. $\mathrm{PH}$ was responsible for the parasite cultivation. All authors read and approved the final manuscript.

Ethics approval and consent to participate

Not applicable

\section{Consent for publication}

Not applicable

\section{Competing interests}

The authors declare that they have no competing interests.

\section{Publisher's Note}

Springer Nature remains neutral with regard to jurisdictional claims in published maps and institutional affiliations.

Received: 30 September 2017 Accepted: 8 January 2018

Published online: 22 January 2018

References

1. Zheng Z, Cheng Z. Advances in molecular diagnosis of malaria. Adv Clin Chem. 2017;80:155-92

2. Cordray MS, Richards-Kortum RR. Emerging nucleic acid-based tests for point-of-care detection of malaria. Am J Trop Med Hyg. 2012;87:223-30.

3. Imai K, Tarumoto N, Misawa K, Runtuwene LR, Sakai J, Hayashida K, et al. A novel diagnostic method for malaria using loop-mediated isothermal amplification (LAMP) and MinION ${ }^{\mathrm{TM}}$ nanopore sequencer. BMC Infect Dis. 2017:17:621.

4. Batista-dos-Santos S, Raiol M, Santos S, Cunha MG, Ribeiro-dos-Santos Â. Real-time PCR diagnosis of Plasmodium vivax among blood donors. Malar J. 2012;11:345

5. Pattaradilokrat S, Tiyamanee W, Simpalipan P, Kaewthamasorn M, Saiwichai T, Li J, et al. Molecular detection of the avian malaria parasite Plasmodium gallinaceum in Thailand. Vet Parasitol. 2015;210:1-9.

6. Snounou G, Viriyakosol S, Jarra W, Thaithong S, Brown KN. Identification of the four human malaria parasite species in field samples by the polymerase 
chain reaction and detection of a high prevalence of mixed infections. Mol Biochem Parasitol. 1993;58:283-92.

7. Brockman A, Paul RE, Anderson TJ, Hackford I, Phaiphun L, Looareesuwan S, et al. Application of genetic markers to the identification of recrudescent Plasmodium falciparum infections on the northwestern border of Thailand. Am J Trop Med Hyg. 1999;60:14-21.

8. Rubio JM, Berzosa PJ, Benito A. Amplified fragment length polymorphism (AFLP) protocol for genotyping the malarial parasite Plasmodium falciparum. Parasitology. 2001;123:331-6.

9. Nyachieo A, VAN Overmeir C, Laurent T, Dujardin JC, D'Alessandro U. Plasmodium falciparum genotyping by microsatellites as a method to distinguish between recrudescent and new infections. Am J Trop Med Hyg. 2005;73:210-3.

10. Pumpaibool T, Arnathau C, Durand P, Kanchanakhan N, Siripoon N, Suegorn A, et al. Genetic diversity and population structure of Plasmodium falciparum in Thailand, a low transmission country. Malar J. 2009:8:155.

11. Ranford-Cartwright LC, Taylor J, Umasunthar T, Taylor LH, Babiker HA, Lell $B$, et al. Molecular analysis of recrudescent parasites in a Plasmodium falciparum drug efficacy trial in Gabon. Trans R Soc Trop Med Hyg. 1997;91:719-24.

12. Borre MB, Dziegiel M, Høgh B, Petersen E, Rieneck K, Riley E, et al. Primary structure and localization of a conserved immunogenic Plasmodium falciparum glutamate rich protein (GLURP) expressed in both the preerythrocytic and erythrocytic stages of the vertebrate life cycle. Mol Biochem Parasitol. 1991;49:119-31.

13. Høgh B, Thompson R, Zakiuddin IS, Boudin C, Borre M. Glutamate rich Plasmodium falciparum antigen (GLURP). Parassitologia. 1993;35:47-50.

14. Paul G, Deshmukh A, Kaur I, Rathore S, Dabral S, Panda A, et al. A novel Pfs38 protein complex on the surface of Plasmodium falciparum bloodstage merozoites. Malar J. 2017;16:79.

15. Theisen M, Cox G, Høgh B, Jepsen S, Vuust J. Immunogenicity of the Plasmodium falciparum glutamate-rich protein expressed by vaccinia virus. Infect Immun. 1994;62:3270-5.

16. Theisen M, Vuust J, Gottschau A, Jepsen S, Høgh B. Antigenicity and immunogenicity of recombinant glutamate-rich protein of Plasmodium falciparum expressed in Escherichia coli. Clin Diagn Lab Immunol. 1995;2:30-4.

17. Dodoo D, Theisen M, Kurtzhals JA, Akanmori BD, Koram KA, Jepsen S, et al. Naturally acquired antibodies to the glutamate-rich protein are associated with protection against Plasmodium falciparum malaria. J Infect Dis. 2000; 181:1202-5.

18. Lusingu JP, Vestergaard LS, Alifrangis M, Mmbando BP, Theisen M, Kitua AY, et al. Cytophilic antibodies to Plasmodium falciparum glutamate rich protein are associated with malaria protection in an area of holoendemic transmission. Malar J. 2005;4:48

19. Nebie I, Diarra A, Ouedraogo A, Soulama I, Bougouma EC, Tiono AB, et al. Humoral responses to Plasmodium falciparum blood-stage antigens and association with incidence of clinical malaria in children living in an area of seasonal malaria transmission in Burkina Faso, West Africa. Infect Immun. 2008;76:759-66.

20. Adu B, Cherif MK, Bosomprah S, Diarra A, Arthur FK, Dickson EK, et al. Antibody levels against GLURP R2, MSP1 block 2 hybrid and AS202.11 and the risk of malaria in children living in hyperendemic (Burkina Faso) and hypo-endemic (Ghana) areas. Malar J. 2016;15:123.

21. Pratt-Riccio LR, Lima-Junior JC, Carvalho LJ, Theisen M, Espíndola-Mendes EC, Santos F, et al. Antibody response profiles induced by Plasmodium falciparum glutamate-rich protein in naturally exposed individuals from a Brazilian area endemic for malaria. Am J Trop Med Hyg. 2005;73:1096-103.

22. Oeuvray C, Theisen M, Rogier C, Trape JF, Jepsen S, Druilhe P. Cytophilic immunoglobulin responses to Plasmodium falciparum glutamate-rich protein are correlated with protection against clinical malaria in Dielmo, Senegal. Infect Immun. 2000;68:2617-20.

23. Kana IH, Adu B, Tiendrebeogo RW, Singh SK, Dodoo D, Theisen M. Naturally acquired antibodies target the glutamate-rich protein on intact merozoites and predict protection against febrile malaria. J Infect Dis. 2017;215:623-30.

24. Theisen M, Soe S, Brunstedt K, Follmann F, Bredmose L, Israelsen H, et al. A Plasmodium falciparum GLURP-MSP3 chimeric protein; expression in Lactococcus lactis, immunogenicity and induction of biologically active antibodies. Vaccine. 2004;22:1188-98.

25. Carvalho $\sqcup$, Alves FA, Bianco C Jr, Oliveira SG, Zanini GM, Soe S, et al. Immunization of Saimiri sciureus monkeys with a recombinant hybrid protein derived from the Plasmodium falciparum antigen glutamate-rich protein and merozoite surface protein 3 can induce partial protection with Freund and Montanide ISA720 adjuvants. Clin Diagn Lab Immunol. 2005;12:242-8.

26. Tamborrini M, Stoffel SA, Westerfeld N, Amacker M, Theisen M, Zurbriggen $\mathrm{R}$, et al. Immunogenicity of a virosomally-formulated Plasmodium falciparum GLURP-MSP3 chimeric protein-based malaria vaccine candidate in comparison to adjuvanted formulations. Malar J. 2011;10:359.

27. Esen M, Kremsner PG, Schleucher R, Gässler M, Imoukhuede EB, Imbault N, et al. Safety and immunogenicity of GMZ2 - a MSP3-GLURP fusion protein malaria vaccine candidate. Vaccine. 2009;27:6862-8.

28. Mordmüller B, Szywon K, Greutelaers B, Esen M, Mewono L, Treut C, et al. Safety and immunogenicity of the malaria vaccine candidate GMZ2 in malaria-exposed, adult individuals from Lambaréné, Gabon. Vaccine. 2010:28:6698-703.

29. Bélard S, Issifou S, Hounkpatin AB, Schaumburg F, Ngoa UA, Esen M, et al. A randomized controlled phase $\mathrm{lb}$ trial of the malaria vaccine candidate GMZ2 in African children. PLoS One. 2011;6:e22525.

30. Jepsen MP, Jogdand PS, Singh SK, Esen M, Christiansen M, Issifou S, et al. The malaria vaccine candidate GMZ2 elicits functional antibodies in individuals from malaria endemic and non-endemic areas. J Infect Dis. 2013;208:479-88.

31. Mamo H, Esen M, Ajua A, Theisen M, Mordmüller B, Petros B. Humoral immune response to Plasmodium falciparum vaccine candidate GMZ2 and its components in populations naturally exposed to seasonal malaria in Ethiopia. Malar J. 2013;12:51.

32. Sirima SB, Mordmüller B, Milligan P, Ngoa UA, Kironde F, Atuguba F, et al. GMZ2 trial study group. A phase $2 b$ randomized, controlled trial of the efficacy of the GMZ2 malaria vaccine in African children. Vaccine. 2016:34:4536-42

33. Takala SL, Plowe CV. Genetic diversity and malaria vaccine design, testing and efficacy: preventing and overcoming 'vaccine resistant malaria'. Parasite Immunol. 2009;31:560-73.

34. Patel P, Bharti PK, Bansal D, Raman RK, Mohapatra PK, Sehgal R, et al. Genetic diversity and antibody responses against Plasmodium falciparum vaccine candidate genes from Chhattisgarh, Central India: implication for vaccine development. PLoS One. 2017;12:e0182674.

35. Kumar D, Dhiman S, Rabha B, Goswami D, Deka M, Singh L, et al. Genetic polymorphism and amino acid sequence variation in Plasmodium falciparum GLURP R2 repeat region in Assam, India, at an interval of five years. Malar J. 2014;13:450.

36. Mohd Abd Razak MR, Sastu UR, Norahmad NA, Abdul-Karim A, Muhammad A, Muniandy PK, et al. Genetic diversity of Plasmodium falciparum populations in malaria declining areas of Sabah, East Malaysia. PLoS One. 2016;11:e0152415.

37. Akter J, Thriemer K, Khan WA, Sullivan DJ Jr, Noedl H, Haque R. Genotyping of Plasmodium falciparum using antigenic polymorphic markers and to study anti-malarial drug resistance markers in malaria endemic areas of Bangladesh. Malar J. 2012;11:386

38. Congpuong K, Hoonchaiyapoom T, Inorn K. Plasmodium falciparum genotype diversity in artemisinin derivatives treatment failure patients along the Thai-Myanmar border. Korean J Parasitol. 2014;52:631-7.

39. Haddad D, Snounou G, Mattei D, Enamorado IG, Fiqueroa J, Ståhl S, et al. Limited genetic diversity of Plasmodium falciparum in field isolates from Honduras. Am J Trop Med Hyg. 1999;60:30-4.

40. Simpalipan P, Pattaradilokrat S, Siripoon N, Seugorn A, Kaewthamasorn M, Butcher RD, et al. Diversity and population structure of Plasmodium falciparum in Thailand based on the spatial and temporal haplotype patterns of the C-terminal 19-kDa domain of merozoite surface protein-1. Malar J. 2014;13:54.

41. Pattaradilokrat S, Sawaswong V, Simpalipan P, Kaewthamasorn M, Siripoon N, Harnyuttanakorn P. Genetic diversity of the merozoite surface protein-3 gene in Plasmodium falciparum populations in Thailand. Malar J. 2016;15:517.

42. Gardner MJ, Hall N, Fung E, White O, Berriman M, Hyman RW, et al. Genome sequence of the human malaria parasite Plasmodium falciparum. Nature. 2002:419:498-511.

43. Hall TA. BioEdit: a user-friendly biological sequence alignment editor and analysis program for Windows 95/98/NT. Nucl Acids Symp Series. 1999:41:95-8.

44. Sievers F, Higgins DG. Clustal omega. Curr Protoc Bioinformatics. 2014; 48:3.13.1-16.

45. Excoffier $L$, Lischer HE. Arlequin suite ver 3.5: a new series of programs to perform population genetics analyses under Linux and Windows. Mol Ecol Resour. 2010;10:564-7. 
46. Librado P, Rozas J. DnaSP v5: a software for comprehensive analysis of DNA polymorphism data. Bioinformatics. 2009;25:1451-2.

47. Rozas J. DNA sequence polymorphism analysis using DnaSP. Methods Mol Biol. 2009;537:337-50.

48. A-Elbasit IE, A-Elgadir TM, Elghazali G, Elbashir MI, Giha HA. Genetic fingerprints of parasites causing severe malaria in a setting of low transmission in Sudan. J Mol Microbiol Biotechnol. 2007;13:89-95.

49. Tajima F. Simple methods for testing the molecular evolutionary clock hypothesis. Genetics. 1993;135:599-607.

50. Fu YX, Li WH. Statistical tests of neutrality of mutations. Genetics. 1993; 133:693-709.

51. Theisen M, Adu B, Mordmüller B, Singh S. The GMZ2 malaria vaccine: from concept to efficacy in humans. Expert Rev Vaccines. 2017;16:907-17.

52. Theisen M, Soe S, Oeuvray C, Thomas AW, Vuust J, Danielsen S, et al. The glutamate-rich protein (GLURP) of Plasmodium falciparum is a target for antibody-dependent monocyte-mediated inhibition of parasite growth in vitro. Infect Immun. 1998;66:11-7.

Submit your next manuscript to BioMed Central and we will help you at every step:

- We accept pre-submission inquiries

- Our selector tool helps you to find the most relevant journal

- We provide round the clock customer support

- Convenient online submission

- Thorough peer review

- Inclusion in PubMed and all major indexing services

- Maximum visibility for your research

Submit your manuscript at www.biomedcentral.com/submit 